\title{
Magnetic Polarizability of a Short Right Circular Conducting Cylinder ${ }^{1}$
}

\author{
T. T. Taylor ${ }^{2}$
}

(August 1, 1960)

\begin{abstract}
The magnetic polarizability tensor of a short right circular conducting cylinder is calculated in the principal axes system with a uniform quasi-static but nonpenetrating applied field. One of the two distinct tensor components is derived from results already obtained in connection with the electric polarizability of short conducting cylinders. The other is calculated to an accuracy of four to five significant figures for cylinders with radius to half-length ratios of $1 / 4,1 / 2,1,2$, and 4 . These results, when combined with the corresponding results for the electric polarizability, are applicable to the problem of calculating scattering from cylinders and to the design of artificial dispersive media.
\end{abstract}

\section{Introduction}

This article considers the problem of finding the magnetic polarizability tensor $\beta_{i j}$ of a short, that is, noninfinite in length, right circular conducting cylinder under the assumption of negligible field penetration. The latter condition can be realized easily with available conducting materials and fields which, although time varying, have wavelengths long compared with cylinder dimensions and can therefore be regarded as quasi-static. The approach of the present article is parallel to that employed earlier [1] ${ }^{3}$ by the author for finding the electric polarizability of short conducting cylinders.

The magnetic polarizability tensor relates the induced magnetic dipole moment $m_{i}$ to the inducing field $B_{j}$ according to the equation

$$
m_{i}=\beta_{i j} B_{j}
$$

with the cylinder situated in free space. The centered coordinate system of principal axes used in [1], in which the $z$-axis coincides with the axis of rotational symmetry, is also employed here. In this system, the equal transverse components $\beta_{x x}$ and $\beta_{y y}$ are denoted $\beta_{t t}$, and the longitudinal component $\beta_{z z}$ is denoted $\beta_{l l}$. In agreement with other conventions of [1], $a$ represents the radius of the cylinder; $b$, the half-length; and $c$, the half-diagonal or distance from center to edge. Rationalized M.K.S. units are used throughout.

The method described has been used successfully for calculating $\beta_{t t}$ for cylinders with $a / b$ ratios of $1 / 4,1 / 2,1,2$, and 4 . For the calculation of $\beta_{l l}$, the following relation, proved in appendix $\mathrm{A}$, is invoked:

$$
\mu_{\mathrm{O}} \beta_{l l}=-\frac{1}{2} \frac{a_{t t}}{\epsilon_{\mathrm{O}}}
$$

where $\alpha_{t t}$ is the transverse electric polarizability treated in [1]. It is interesting that eq. (2) is valid not only for circular cylinders but for all conducting solids of revolution. Both $\beta_{\imath \imath}$ and $\beta_{t t}$ are negative, indicating that an artificial medium composed of short cylinder elements arranged in a lattice will be diamagnetic.

In the transverse case, where no simple relationship such as (2) is known to exist, the prob-

\footnotetext{
1 This invited paper is based upon a portion of a thesis which has been accepted by the faculty of the California Institute of Technology in partial fulfillment of the requirements for the degree of Doctor of Philosophy.

2 University of California, Riverside.

${ }^{3}$ Figures in brackets indicate the literature references at the end of this paper.
} 
lem which presents itself is that of finding, in the external space, a static magnetic field which has no normal component at the surface of the cylinder and which reduces to a uniform field at infinity. With such a field there is associated physically a surface current density $\mathbf{j}$ on the cylinder and a field free condition in the interior. This surface current is related to the field values at the surface by:

$$
\mu_{\mathrm{o}} \mathbf{j}=\mathbf{e}_{n} \times \mathbf{B},
$$

where $\mathbf{e}_{n}$ is the outward normal, and it gives rise to the induced magnetic dipole moment:

$$
\mathbf{m}=\frac{1}{2} \int_{s} \mathbf{r} \times \mathbf{j} d s .
$$

As with the corresponding problem involving electric polarizability, there is no known coordinate system in which a short cylinder can be treated by the conventional method of separating Laplace's equation and a different method, involving arbitrarily good approximations to the current distribution function, must be employed.

In a manner analogous to that of [1], let the cylinder be regarded merely as a geometrical construct to which the surface current distribution is firmly affixed. If the uniform (applied) component of the magnetic field is subtracted, the remaining field will be that due to the current system on the cylinder acting alone; it will be characterized by a dipole-like external field and a perfectly uniform internal field which is equal and opposite to the applied field. The problem may therefore be restated in terms of finding, on a finite cylindrical surface with closed plane ends, a surface current distribution which, when acting alone, generates a uniform magnetic field in the interior. The technique employed here makes use of this viewpoint and consists in setting up, on the surface, a current density function which is completely determined by a finite number of parameters, then solving for the values of these parameters so as to obtain maximal uniformity of the current field or, alternatively, maximal cancellation of the applied field, within.

The research reported here, like that related to the electric polarizability, was performed under the direction of Prof. W. R. Smythe. The author is indebted to him for many valuable discussions and to the personnel of the California Institute of Technology Computing Center for their cooperation.

\section{The Transverse Problem}

In this problem, let a uniform magnetic field of magnitude $B$ be applied in the positive $y$-direction. The induced surface current density $\mathbf{j}$ may be regarded as equal to $\mathbf{J} \xi$ where $\xi$ is the thickness of an infinitesmal surface layer in which the current is confined and $\mathbf{J}$ is the value of the conventional current density vector averaged over the thickness of this layer. In the quasi-static case, $\mathbf{J}$ is solenoidal and can be regarded as the curl of an equivalent averaged magnetization $\mathbf{M}$ which is also confined to the infinitesmal surface layer and which is normal to the surface at all points. On the side of the cylinder, let

$$
\frac{\mu_{0} M_{\rho} \xi}{B}=-a F(z / b) \sin \phi
$$

and on the upper end,

$$
\frac{\mu_{0} M_{z} \xi}{B}=-a^{\prime} G(\rho / a) \sin \phi
$$

where $F$ and $G$ are dimensionless functions as yet to be determined. The configuration just described constitutes a nonuniform magnetic shell, that is a magnetic shell [2, p. 261] associated with a distributed current system. 
Evidently the surface currents on the side are given by

$$
\begin{aligned}
& \frac{\mu_{0} j_{z s}}{B}=F(z / b) \cos \phi, \\
& \frac{\mu_{0} j_{\phi s}}{B}=-\frac{a}{b} F^{\prime}(z / b) \sin \phi,
\end{aligned}
$$

where the prime denotes differentiation with respect to the dimensionless argument $z / b$. The surface currents on the upper end are

$$
\begin{aligned}
& \frac{\mu_{0} j_{\rho e}}{B}=-\frac{a}{\rho} G(\rho / a) \cos \phi ; \\
& \frac{\mu_{0} j_{\phi e}}{B}=G^{\prime}(\rho / a) \sin \phi \cdot
\end{aligned}
$$

The current distribution on the lower end is equal and opposite to that on the upper end. Typical forms for the functions involved in eqs (7) through (10) are illustrated in figure 1; a sketch of the actual lines of current flow is presented in figure 2.
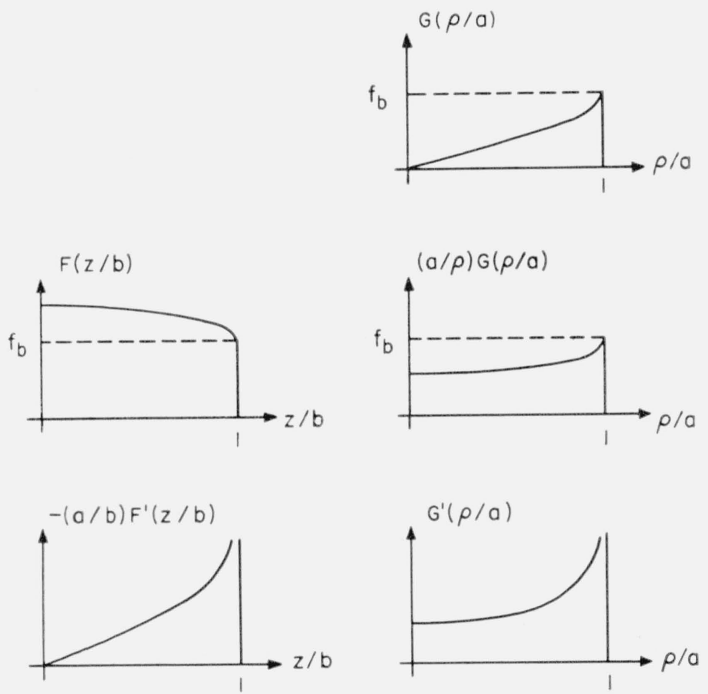

FIguRE 1. Typical forms for the functions describing the equivalent magnetic shell and the current system.

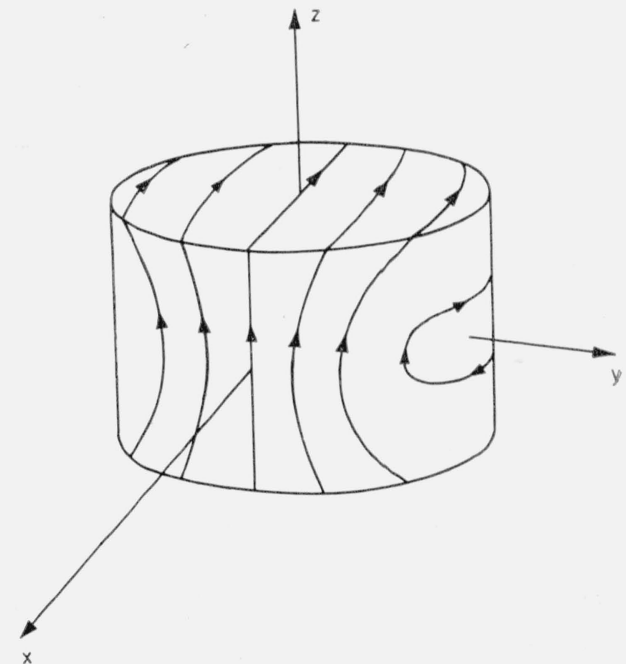

FIGURE 2. Sketch of the current system.

The asymptotic behavior of current and field near the edge of the cylinder is determined by the fact that, locally, the edge resembles a configuration consisting of two infinite perfectly conducting planes intersecting at right angles with the field restricted to the outer (larger) of the two angular domains. Such a configuration is a familiar one in two-dimensional potential theory. In the case at hand, two mutually perpendicular components of magnetic field are distinguished: $(a)$ the component which is everywhere parallel to the edge, and $(b)$ the component which bends around the edge. Each of these field components has associated with it a surface current obeying eq (3). The component of magnetic field parallel to the edge is locally uniform in the outer space and is of no further interest in the present discussion. The surface value of the component which bends around the edge, however, increases as the edge is approached and is in fact proportional to $l^{-1 / 3}$ where $l$ is the perpendicular distance from 
the edge to the surface point in question. This dependence, which is the same as that of the electrostatic charge density on a similar freely charged configuration, is obtained by means of the Schwarz-Christoffel transformation. The associated component of surface current is, of course, parallel to the edge and everywhere proportional to the field. On the cylinder, then, the $\phi$-components of current, and hence the functions $F^{\prime}$ and $G^{\prime}$ must be asymptotic to constants times $l^{-1 / 3}$ as $l$ tends to zero. This, in turn, imposes certain requirements upon $F$ and G. Quantitatively,

$$
\begin{array}{rlrl}
F(z / b) & \sim C_{1}+C_{2}(b-z)^{2 / 3} & & \text { as } \quad z \rightarrow b ; \\
G(\rho / a) & \sim C_{3}-C_{4}(a-\rho)^{2 / 3} & \text { as } \quad \rho \rightarrow a ; \\
-\frac{a}{b} F^{\prime}(z / b) & \sim \frac{2}{3} a C_{2}(b-z)^{-1 / 3} & & \text { as } \quad z \rightarrow b ; \\
G^{\prime}(\rho / a) & \sim \frac{2}{3} a C_{4}(a-\rho)^{-1 / 3} & & \text { as } \quad \rho \rightarrow a .
\end{array}
$$

It is seen that two edge conditions, $C_{3}=C_{1}$ and $C_{4}=C_{2}$, must be satisfied. The former of these insures continuity of current flow across the edge of the cylinder, the latter that the $\phi$ components on side and end match one another as both tend to infinity at the edge.

The functions $F$ and $G$ are represented by finite weighted sums involving the unknown coefficients $f_{b}, f_{m}, g_{b}$, and $g_{m}$, and the $\bar{\psi}_{m}$ functions defined in [1]. Note that the parity parameters are chosen so as to make $F$ even and $G$ odd and that the parameter which controls the singularity is given the value $2 / 3$.

Thus,

$$
F(z / b)=\left\{\begin{array}{ll}
f_{b}+\sum_{m=0}^{N_{\mathrm{s}}-1} f_{m} \bar{\psi}_{m}\left(\frac{1}{2}, 0, \frac{2}{3}, \frac{z}{b}\right), & |z| \leq b, \\
0, & |z|>b,
\end{array}\right\}
$$

and

$$
G(\rho / a)=\left\{\begin{array}{ll}
g_{b} \frac{\rho}{a}+\sum_{m=0}^{N_{\mathrm{e}}-1} g_{m} \bar{\psi}_{m}\left(1,1, \frac{2}{3}, \frac{\rho}{a}\right) & \rho \leq a \\
0 & \rho>a
\end{array}\right\}
$$

The constant terms

$$
\begin{aligned}
& f_{b}=f_{b} \bar{\psi}_{0}\left(\frac{1}{2}, 0,0, \frac{z}{b}\right), \\
& g_{b} \frac{\rho}{a}=g_{b} \bar{\psi}_{c}\left(1,1,0, \frac{\rho}{a}\right),
\end{aligned}
$$

are called the "basic" terms as in [1]. Together they constitute a very simple current system in which the flow takes place in plane rectangular loops around the cylinder, the plane of each loop being parallel to the $x z$-plane of figure 2. Superposed upon this basic flow pattern is another pattern described by the nonbasic (summed) terms in (15) and (16). The addition of this pattern gives a gentle curvature to the current lines and introduces the necessary singular behavior at the edges. Clearly the first edge condition demands that $g_{b}=f_{b}$ and henceforth the single symbol $f_{b}$ will be used as the coefficient of the basic term in both $F$ and $G$.

In relating the self field of the current system to $\mathbf{M} \xi$, a magnetic scalar potential $U$, defined such that $\mathbf{B}=-\nabla U$, will be used. The boundary conditions at the magnetic shell are the duals of the corresponding boundary conditions for the electric potential at a charged surface, that is, the normal gradients on the two sides of the shell are equal but the potential values differ by the local intensity of the magnetization. Quantitatively, if I and II are two adjoining 
regions separated by a magnetic shell, and if $\mathbf{e}_{n}$ is a unit vector pointing from I into II, then

$$
\begin{gathered}
\frac{\partial U_{\mathrm{II}}}{\partial n}-\frac{\partial U_{1}}{\partial n}=0 ; \\
U_{\mathrm{II}}-U_{\mathrm{I}}=\mu_{0} M_{n} \xi .
\end{gathered}
$$

Application of these boundary conditions and of other well-known procedures of potential theory leads to the following expression for the potential in the interior of the cylinder due to the current system on the side:

$$
\frac{U_{s}(\rho, \phi, z)}{c B}=\frac{-a}{c} \int_{0}^{\infty}\left[\frac{2}{\pi} \int_{0}^{b} F\left(z^{\prime} / b\right) \cos k z^{\prime} d z^{\prime}\right] k a K_{1}^{\prime}(k a) I_{1}(k \rho) \cos k z d k \sin \phi .
$$

The Fourier cosine transform of the $F$ function, enclosed in the square brackets above, is easily expressed in terms of Bessel functions with the aid of eq (A9) in [1]. The function $I_{1}(k \rho) \cos$ $k z \sin \phi$, which is regular in a neighborhood of the origin, may be represented in a spherical harmonic expansion about the origin. Thus (21) becomes

$$
\begin{aligned}
\frac{U_{s}(r, \theta, \phi)}{c B}=\sum_{p=0}^{\infty} \frac{-a b}{c}\left(\frac{2}{\pi}\right)^{1 / 2} \int_{0}^{\infty}\left[f_{b} \frac{J_{1 / 2}(k b)}{(k b)^{1 / 2}}+\sum_{m=0}^{N_{s}-1} f_{m} \frac{(-1)^{m} J_{2 m+1 / 2+\nu^{\prime}}(k b)}{(k b)^{1 / 2+\nu^{\prime}}}\right] \\
k a K_{1}^{\prime}(k a)(k c)^{2 p+1} d k \frac{(-1)^{p}}{(2 p+2) !}\left(\frac{r}{2}\right)^{2 p+1} P_{2 p+1}^{1}(\cos \theta) \sin \phi .
\end{aligned}
$$

Here and elsewhere, the singularity parameter $\nu^{\prime}$ for the nonbasic terms is understood to be equal to $2 / 3$. The spherical harmonic expansion of $(22)$ converges for all $r<a$.

With reference to the ends of the cylinder, the interior potential generated by the current distributions on the two ends is given by

$$
\frac{U_{e}(\rho, \phi, z)}{c B}=\frac{a}{c} \int_{0}^{\infty}\left[\frac{1}{a} \int_{0}^{a} G\left(\rho^{\prime} / a\right) J_{1}\left(k \rho^{\prime}\right) \rho^{\prime} d \rho^{\prime}\right] k a e^{-k b} J_{1}(k \rho) \cosh k z d k \sin \phi .
$$

This, in turn, may also be expressed as an expansion in spherical harmonics which converges for all $r<b$ :

$$
\begin{aligned}
\frac{U_{e}(r, \theta, \phi)}{c B}=\sum_{p=0}^{\infty} \frac{a^{2}}{c} \int_{0}^{\infty}\left[f_{b} \frac{J_{2}(k a)}{(k a)}+\sum_{m=0}^{N_{e}-1} g_{m} \frac{(-1)^{m} \boldsymbol{J}_{2 m+2+\nu^{\prime}}(k a)}{(k a)^{1+\nu^{\prime}}}\right] & k a e^{-k b}(k c)^{2 p+1} d k \frac{1}{(2 p+2) !}\left(\frac{r}{c}\right)^{2 p+1} P_{2 p+1}^{1}(\cos \theta) \sin \phi .
\end{aligned}
$$

Evidently the sum of (22) and (24) constitutes an expansion of the interior potential due to the total current distribution, convergent within the largest sphere which can be inscribed in the cylinder. Such an expansion may also be expressed in the form

$$
\frac{U(r, \theta, \phi)}{c B}=\sum_{p=0}^{\infty}\left[f_{b} Z_{p}^{s b}+\sum_{m=0}^{N_{s}-1} f_{m} Z_{p}^{s m}+f_{b} Z_{p}^{e b}+\sum_{m=0}^{N_{e}-1} g_{m} Z_{p}^{e m}\right]\left(\frac{r}{c}\right)^{2 p+1} P_{2 p+1}^{1}(\cos \theta) \sin \phi,
$$

where the Z's are obtained from (22) and (24) by integrating [3, p. 137] over $k$. These Z's become the matrix elements in a system of simultaneous equations which may be solved for the $f_{b}, f_{m}$, and $g_{m}$ such that the coefficient of $(r / c)^{2 p+1} P_{2 p+1}^{1}(\cos \theta) \sin \phi$ will be unity for $p=0$ and zero for as many $p>0$ as possible. The second edge condition, also included in the system of simultaneous equations, is simply

$$
\sum_{m=0}^{N_{s}-1} f_{m}+\left(\frac{a}{b}\right)^{-2 / 3} \sum_{m=0}^{N_{e}-1} g_{m}=0
$$


If one makes use of the fact that $-k a K_{1}^{\prime}(k a)=k a K_{0}(k a)+K_{1}(k a)$, the expression for the matrix element $Z_{P}^{s m}$ becomes

$$
Z_{p}^{s m}=\frac{a b}{c}\left(\frac{2}{\pi}\right)^{1 / 2} \frac{(-1)^{m+p}}{(2 p+2) !} \int_{0}^{\infty}\left[k a K_{0}(k a)+K_{1}(k a)\right] \frac{J_{2 m+\frac{1}{2}+v^{\prime}}(k b)}{(k b)^{1 / 2+\nu^{\prime}}}(k c)^{2 p+1} d k .
$$

Integration yields the following result:

$$
\begin{aligned}
Z_{p}^{s m}= & \frac{(-1)^{m+p} 2^{2 p+1-\nu^{\prime}} \Gamma\left(m+p+\frac{3}{2}\right) \Gamma\left(m+p+\frac{1}{2}\right)}{\Gamma\left(\frac{1}{2}\right) \Gamma(2 p+3) \Gamma\left(2 m+\frac{3}{2}+\nu^{\prime}\right)} \frac{a^{2}}{b c}\left(\frac{b}{c}\right)^{2 m+2}\left[\left(m+p+\frac{1}{2}\right)\right. \\
& \left.{ }_{2} F_{1}\left(m+p+\frac{3}{2}, m-p+\nu^{\prime} ; 2 m+\frac{3}{2}+\nu^{\prime} ; \frac{b^{2}}{c^{2}}\right)+\frac{1}{2}{ }_{2} F_{1}\left(m+p+\frac{3}{2}, m-p+1+\nu^{\prime} ; 2 m+\frac{3}{2}+\nu^{\prime} ; \frac{b^{2}}{c^{2}}\right)\right] .
\end{aligned}
$$

The matrix element $Z_{P}^{e m}$ is simpler:

$$
Z_{p}^{e m}=\frac{a^{2}}{c} \frac{(-1)^{m}}{(2 p+2) !} \int_{0}^{\infty} e^{-k b} \frac{J_{2 m+2+\nu^{\prime}}(k a)}{(k a)^{1+\nu \prime}}(k c)^{2 p+1} k a d k .
$$

This becomes

$$
\begin{aligned}
& Z_{p}^{e m}=\frac{(-1)^{m} 2^{2 p+1-\nu^{\prime}} \Gamma\left(m+p+\frac{5}{2}\right) \Gamma(m+p+2)}{\Gamma\left(\frac{1}{2}\right) \Gamma(2 p+3) \Gamma\left(2 m+3+\nu^{\prime}\right)} \frac{b}{c}\left(\frac{a}{c}\right)^{2 m+4} \\
& { }_{2} F_{1}\left(m+p+\frac{5}{2}, m-p+1+\nu^{\prime} ; 2 m+3+\nu^{\prime} ; \frac{a^{2}}{c^{2}}\right) .
\end{aligned}
$$

The basic matrix elements, $Z_{p}^{s b}$ and $Z_{p}^{e b}$, are obtained by setting both $m$ and $\nu^{\prime}$ equal to zero in the preceding formulas.

Thus,

$$
\begin{gathered}
Z_{p}^{s b}=\frac{(-1)^{p} 2^{2 p+1} \Gamma\left(p+\frac{3}{2}\right) \Gamma\left(p+\frac{1}{2}\right)}{\Gamma\left(\frac{1}{2}\right) \Gamma(2 p+3) \Gamma\left(\frac{3}{2}\right)} \frac{a^{2} b}{c^{3}}\left[\left(p+\frac{1}{2}\right)\right. \\
\left.{ }_{2} F_{1}\left(p+\frac{3}{2},-p ; \frac{3}{2} ; \frac{b^{2}}{c^{2}}\right)+\frac{1}{2}{ }_{2} F_{1}\left(p+\frac{3}{2},-p+1 ; \frac{3}{2} ; \frac{b^{2}}{c^{2}}\right)\right] ; \\
Z_{p}^{e b}=\frac{2^{2 p+1} \Gamma\left(p+\frac{5}{2}\right) \Gamma(p+2)}{\Gamma\left(\frac{1}{2}\right) \Gamma(2 p+3) \Gamma(3)} \frac{a^{4} b}{c^{5}}{ }_{2} F_{1}\left(p+\frac{5}{2},-p+1 ; 3 ; \frac{a^{2}}{c^{2}}\right) .
\end{gathered}
$$

Using the appropriate formulas for analytic continuation [4, p. 108], one obtains

$$
\begin{aligned}
{ }_{2} F_{1}\left(p+\frac{5}{2},-p+1 ; 3 ; \frac{a^{2}}{c^{2}}\right)=\frac{\Gamma(3) \Gamma\left(-\frac{1}{2}\right)}{\Gamma\left(-p+\frac{1}{2}\right) \Gamma(p+2)}{ }_{2} F_{1}\left(p+\frac{5}{2},-p+1 ; \frac{3}{2} ; \frac{b^{2}}{c^{2}}\right) \\
\quad+\frac{c}{b} \frac{\Gamma(3) \Gamma\left(\frac{1}{2}\right)}{\Gamma\left(p+\frac{5}{2}\right) \Gamma(-p+1)}{ }_{2}^{2} F_{1}\left(-p+\frac{1}{2}, p+2 ; \frac{1}{2} ; \frac{b^{2}}{c^{2}}\right) .
\end{aligned}
$$

By [4, p. 3],

$$
\frac{\Gamma\left(-\frac{1}{2}\right)}{\Gamma\left(-p+\frac{1}{2}\right)}=\frac{-(-1)^{p} \Gamma\left(p+\frac{1}{2}\right)}{\Gamma\left(\frac{3}{2}\right)} \text {. }
$$

The second term in (33) vanishes unless $p=0$, in which case the hypergeometric function is easily summed:

$$
{ }_{2} F_{1}\left(\frac{1}{2}, 2 ; \frac{1}{2} ; \frac{b^{2}}{c^{2}}\right)=\left(1-\frac{b^{2}}{c^{2}}\right)^{-2}=\left(\frac{c}{a}\right)^{4}
$$

Let the symbol $\delta_{o p}$ be equal to unity for $p=0$ and to zero for $p \neq 0$; then

$$
Z_{p}^{e b}=\delta_{0 p}-\frac{(-1)^{p} 2^{2 p+1} \Gamma\left(p+\frac{5}{2}\right) \Gamma\left(p+\frac{1}{2}\right)}{\Gamma\left(\frac{1}{2}\right) \Gamma(2 p+3) \Gamma\left(\frac{3}{2}\right)} \frac{a^{4} b}{c^{5}}{ }_{2} F_{1}\left(p+\frac{5}{2},-p+1 ; \frac{3}{2} ; \frac{b^{2}}{c^{2}}\right) .
$$


In (25), the total coefficient of $f_{b}$ is the sum of $Z_{P}^{s b}$ and $Z_{P}^{e b}$. This sum is denoted by $Z_{p}^{b}$ and is given by

$$
\begin{aligned}
Z_{p}^{b}=\delta_{n_{p}}+\frac{(-1)^{p} 2^{2 p+1} \Gamma\left(p+\frac{3}{2}\right) \Gamma\left(p+\frac{1}{2}\right)}{\Gamma\left(\frac{1}{2}\right) \Gamma(2 p+3) \Gamma\left(\frac{3}{2}\right)} \frac{a^{2} b}{c^{3}} & {\left[\left(p+\frac{1}{2}\right){ }_{2} F_{1}\left(p+\frac{3}{2},-p ; \frac{3}{2} ; \frac{b^{2}}{c^{2}}\right)\right.} \\
+ & \left.\frac{1}{2} F_{1} F_{1}\left(p+\frac{3}{2},-p+1 ; \frac{3}{2} ; \frac{b^{2}}{c^{2}}\right)-\frac{a^{2}}{c^{2}}\left(p+\frac{3}{2}\right)_{2} F_{1}\left(p+\frac{5}{2},-p+1 ; \frac{3}{2} ; \frac{b^{2}}{c^{2}}\right)\right] .
\end{aligned}
$$

Using the third Gauss relationship, as listed in [4, p. 103]:

$$
Z_{p}^{b}=\delta_{0 p}-\frac{(-1)^{p} 2^{2 p} \Gamma\left(p+\frac{3}{2}\right) \Gamma\left(p+\frac{1}{2}\right)}{\Gamma\left(\frac{1}{2}\right) \Gamma(2 p+3) \Gamma\left(\frac{3}{2}\right)} \frac{a^{2} b}{c^{3}}{ }_{2} F_{1}\left(p+\frac{3}{2},-p+1 ; \frac{3}{2} ; \frac{b^{2}}{c^{2}}\right)
$$

This has an even simpler form, obtained by an elementary transformation of the hypergeometric function and a simplification of the gamma function multiplier:

$$
\mathrm{Z}_{p}^{b}=\delta_{0 p}-\frac{(-1)^{p} \Gamma\left(p+\frac{1}{2}\right)}{2 \Gamma\left(\frac{1}{2}\right) \Gamma(p+2)} \frac{b}{c}{ }_{2} F_{1}\left(p+\frac{1}{2},-p ; \frac{3}{2} ; \frac{b^{2}}{c^{2}}\right) .
$$

The three formulas, (28), (30), and (39), contain the information necessary for a numerical calculation of the matrix elements. If both the electric and the magnetic polarizabilities are being calculated for the same cylinder, much labor can be saved by comparing the expressions for the matrix elements in this paper with those in [1] and exploiting the similarities.

The magnetic dipole moment, given by eq (4), has only a $y$-component,

$$
m_{y}=\frac{1}{2} \int_{-b}^{b} \int_{0}^{2 \pi}\left(-z j_{\phi s} \sin \phi-a j_{z s} \cos \phi\right) a d \phi d z+\int_{0}^{a} \int_{0}^{2 \pi} b\left(j_{\rho e} \cos \phi-j_{\phi e} \sin \phi\right) d \phi \rho d \rho .
$$

Using expressions (7) through (10),

$$
\begin{gathered}
\frac{\mu_{0} m_{y}}{B}=\frac{1}{2} \int_{-b}^{b} \int_{0}^{2 \pi}\left(z \frac{a}{b} F^{\prime} \sin ^{2} \phi-a F \cos ^{2} \phi\right) a d \phi d z+\int_{0}^{a} \int_{0}^{2 \pi} b\left(-\frac{a}{\rho} G \cos ^{2} \phi-G^{\prime} \sin ^{2} \phi\right) d \phi \rho d \rho \\
\frac{\mu_{0} m_{y}}{B}=\pi a^{2} t\left[\int_{0}^{1} \cdots\left(u F^{\prime}-F\right) d u-\int_{0}^{1}\left(G+u G^{\prime}\right) d u\right] .
\end{gathered}
$$

By parts,

$$
\frac{\mu_{0} m_{y}}{B}=\pi a^{2} b\left\{[u F]_{0}^{1}-2 \int_{0}^{1} F d u-[u G]_{0}^{1}\right\}
$$

The first edge condition requires that $F(1)=G(1)$; hence,

$$
\frac{\mu_{0} m_{y}}{B}=-2 \pi a^{2} b \int_{0}^{1} F d u \text {. }
$$

The quantity $m_{y} / B$ is the transverse polarizability and the geometrical volume $v_{0}$ is easily recognized. The orthogonality relationships given in [1] aid in the integration of $F$, yielding the following formula for the polarizability:

$$
\frac{\mu_{0} \beta_{t t}}{v_{0}}=-\left[f_{b}+\frac{\Gamma\left(\frac{1}{2}\right)}{2^{5 / 3} \Gamma\left(\frac{13}{6}\right)} f_{0}\right]
$$




\section{Results}

The two distinct tensor components of the magnetic polarizability are given in table 1 . Actual values of the expansion coefficients for the $F$ and $G$ functions, which describe the surface current distribution via the equivalent magnetic shell for the transverse case, are included in appendix B. These data, upon which the $\beta_{t t}$ results of table 1 are based, were obtained by solving sets of 18 simultaneous equations. Experimentation with fewer equations has led the author to the conclusion that the accuracy is not quite as good as that obtained for the electric polarizability in [1]. In other words, the $\beta_{t t}$ figures have a probable error of about 2 units in the least significant digit for $a / b$ equal to unity and about 10 units in this digit for $a / b$ equal to 4 or to $\frac{1 / 4}{4}$. The $\beta_{l l}$ figures, on the other hand, were obtained from the electric polarizabilities simply by the application of eq (2), and therefore enjoy the same accuracy belonging to the latter.

TABLE 1

\begin{tabular}{|c|c|c|}
\hline$a / b$ & $\mu_{0} \boldsymbol{\beta}_{l l} / v_{0}$ & $\mu_{0} \boldsymbol{\beta}_{t t} / v_{0}$ \\
\hline $\begin{array}{l}0 \\
1 / 4 \\
1 / 2 \\
1 \\
2 \\
4 \\
\infty\end{array}$ & $\begin{array}{l}-1.0000 \\
-1.1575 \\
-1.3057 \\
-1.5853 \\
-2.1087 \\
-3.0907 \\
-\infty\end{array}$ & $\begin{array}{l}-2.0000 \\
-1.8506 \\
-1.7351 \\
-1.5795 \\
-1.4140 \\
-1.2716 \\
-1.0000\end{array}$ \\
\hline \multicolumn{3}{|c|}{$v_{0}=2 \pi a^{2} b$} \\
\hline
\end{tabular}

As an additional check upon the numerical calculations in the transverse problem, a procedure analogous to that employed in [1] was used. Consider the total field $\mathbf{B}_{t}$ which is the sum of the applied field and the field due to the current system on the cylinder. A normal component $B_{t n}$ of the total field at any point on the surface of the cylinder indicates that an error is present. If $n$ represents distance away from the surface in the outward normal direction at a particular surface point, one may calculate the displacement of the surface $\Delta n$ which, considering the local value of the normal field gradient, would reduce the normal field component to zero. In other words, let the following equation be solved for $\Delta n$ :

$$
B_{t n}+\frac{\partial B_{t n}}{\partial n} \Delta n=0
$$

This $\Delta n$ may then be compared with the gross dimensions of the cylinder. The local field gradient $\partial B_{t n} / \partial n$ depends upon the current distribution at the point in question and may be calculated by appealing to eq (3) and to the solenoidal character of $\mathbf{B}_{t}$. To first order, one has for any point on the side of the cylinder

$$
\begin{aligned}
& \frac{\partial B_{t \rho}}{\partial \rho}=\mu_{0}\left(-\frac{1}{a} \frac{\partial j_{z s}}{\partial \phi}+\frac{\partial j_{\phi s}}{\partial z}\right) \\
& \frac{\partial B_{t \rho}}{\partial \rho}=\frac{B \sin \phi}{a}\left(F-\frac{a^{2}}{b^{2}} F^{\prime \prime}\right) .
\end{aligned}
$$

Let the $\rho$-component of the field due to the current system alone be denoted $B_{\rho}$. Then $B_{t \rho}=B_{\rho}+B \sin \phi, \quad \Delta n=\Delta \rho$, and one obtains for the latter,

$$
\frac{\Delta \rho}{a}=\frac{\frac{B_{\rho}}{B \sin \phi}+1}{-F+\frac{a^{2}}{b^{2}} F^{\prime \prime}} .
$$


The quantities $B_{\rho}, F, F^{\prime \prime}$ and finally $\Delta \rho / a$ were calculated for $z=0$, that is for points on the "equator" of the cylinder; hence the term "equator check" in appendix B.

The same method applied to the upper end of the cylinder yields

$$
\begin{aligned}
& \frac{\partial B_{t z}}{\partial z}=\mu_{0}\left(-\frac{j_{\phi e}}{\rho}-\frac{\partial j_{\phi e}}{\partial \rho}+\frac{1}{\rho} \frac{\partial j_{\rho e}}{\partial \phi}\right), \\
& \frac{\partial B_{t z}}{\partial z}=\frac{B \sin \phi}{a}\left(-\frac{G^{\prime}}{u}-G^{\prime \prime}+\frac{G}{u^{2}}\right),
\end{aligned}
$$

where $u$ represents $\rho / a$. Substitution of the expansion $A_{1} u+A_{3} u^{3}+A_{5} u^{5}+\ldots$ for $G$, which is known to be an odd function, gives

$$
\frac{\partial B_{t z}}{\partial z}=\frac{B \sin \phi}{a}\left(-8 A_{3} u-24 A_{5} u^{3}+\ldots\right) .
$$

Since both sides of (52) vanish at $\rho=0$, that is at the "pole" of the cylinder, one must differentiate with respect to $\rho$ in order to obtain something useful. Thus,

$$
\frac{\partial^{2} B_{t z}}{\partial \rho \partial z}=\frac{B \sin \phi}{a^{2}}\left(-8 A_{3}\right)=\frac{-4 B \sin \phi}{3 a^{2}} G^{\prime \prime \prime}(0) .
$$

Furthermore, for any point on the upper end, $B_{t z}$ is simply $B_{z}$, the field due to the current system alone. Finally,

$$
\frac{\Delta z}{b}=\frac{3 a^{2}}{4 b G^{\prime \prime \prime}(0)} \frac{\partial}{\partial \rho}\left(\frac{B_{z}}{B \sin \phi}\right)
$$

Values of $\partial B_{z} / \partial \rho, G^{\prime \prime \prime}$, and $\Delta z / b$ were calculated for the pole; data are given under the heading of "pole check" in appendix B. Generally speaking, the pole checks are satisfactory but are not as good as the corresponding checks in [1].

\section{Appendix A: Relation Between the Longitudinal Magnetic and the Trans- verse Electric Polarizabilities of a Conducting Solid of Revolution}

Let figure 3 illustrate a typical solid of revolution formed by rotating the curve $\rho=\rho(z)$ about the z-axis.

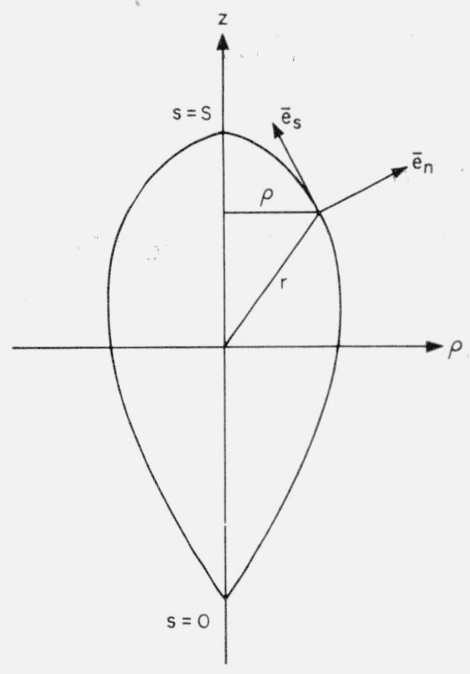

FIGURE 3. Cross section of a typical solid of revolution shouring coordinates and unit vectors. 
Let the unit vectors $\mathbf{e}_{s}$ and $\mathbf{e}_{n}$ point along the tangent and the outward normal of this curve, respectively. The variable $s$, associated with $\mathbf{e}_{s}$, measures distance along the curve and encompasses the solid as it varies over the interval $0 \leq s \leq S$. It is sufficient if $\rho(z)$ has a uniquely defined tangent and is normal at almost every point in this interval.

If the applied fields consist of a uniform magnetic field $B$ in the $z$-direction and a uniform electric field $E$ in the $x$-direction, the following is to be proved:

$$
\frac{\mu_{0} m_{z}}{B}=-\frac{1}{2} \frac{p_{x}}{\epsilon_{0} E}
$$

In the electric case, the scalar potential satisfies the conditions:

$$
\begin{aligned}
V & =-E f_{1}(\rho, z) \cos \phi ; \\
f_{1} & =0 \quad \text { on the surface of the solid; } \\
f_{1} & \sim \rho \quad \text { at infinity; } \\
\nabla^{2} V & =0 .
\end{aligned}
$$

The magnetic case, on the other hand, is analyzed with the aid of the vector potential which, by symmetry, has only a $\phi$-component. This component must vanish at $s=0, s=S$, and at all intermediate surface points; otherwise there would be a normal component of magnetic field. at the surface. One finds that

$$
\left.\begin{array}{rl}
\mathbf{A} & =\mathbf{e}_{\phi} \frac{B}{2} f_{2}(\rho, z) ; \\
f_{2} & =0 \quad \text { on the surface of the solid; } \\
f_{2} & \sim \rho \quad \text { at infinity; } \\
\nabla^{2} \mathbf{A} & =0 .
\end{array}\right\}
$$

Application of the Laplacian operator to either $\cos \phi f_{1}$ or to $\mathbf{e}_{\phi} f_{2}$ yields the same equation in $\rho$ and $z$. In other words,

$$
\left\{\frac{\partial^{2}}{\partial \rho^{2}}+\frac{1}{\rho} \frac{\partial}{\partial \rho}-\frac{1}{\rho^{2}}+\frac{\partial^{2}}{\partial z^{2}}\right\}\left\{\frac{f_{1}}{f_{2}}\right\}=0 .
$$

Since $f_{1}$ and $f_{2}$ obey the same differential equation, have the same boundary conditions at the surface of the solid, and have identical asymptotic behavior at infinity, it is clear that $f_{1}=f_{2}=f$. The surface charge density in the electric case is

$$
\sigma=-\epsilon_{0} \mathbf{e}_{n} \cdot \nabla V=\epsilon_{0} E \cos \phi\left(\mathbf{e}_{n} \cdot \nabla f\right),
$$

and it is easily shown that the $x$-component of the induced electric dipole moment is

$$
p_{x}=\epsilon_{0} E \pi \int_{0}^{s}\left(\mathbf{e}_{n} \cdot \nabla f\right) \rho^{2} d s .
$$

The surface current in the magnetic case as given by (3) is:

$$
\mathbf{j}=\frac{1}{\mu_{0}} \mathbf{e}_{n} \times(\nabla \times \mathbf{A})=\frac{B}{2 \mu_{0}} \mathbf{e}_{n} \times\left(\nabla \times \mathbf{e}_{\phi} f\right) .
$$

On the surface, where $f=0$, this reduces to the following: 


$$
\mathbf{j}=-\frac{B}{2 \mu_{0}} \mathbf{e}_{\phi}\left(\mathbf{e}_{n} \cdot \nabla f\right)
$$

and the magnetic dipole moment is obviously

$$
m_{z}=-\frac{B}{\mu_{0}} \frac{\pi}{2} \int_{0}^{S}\left(\mathbf{e}^{n} \cdot \nabla f\right) \rho^{2} d s .
$$

A comparison of (A9) with (A6) shows that the asserted relation (A1) is true.

\section{Appendix B: Values of the Expansion Coefficients for the Functions F and G}

Values of the expansion coefficients for the functions $F$ and $G$ and the results of the checking procedure at pole and equator are given in tables 2 through 6 . The notation " $A(p)$ " means "A times $10^{p}$."

\begin{tabular}{|c|c|c|}
\hline \multicolumn{3}{|c|}{$a / b=1 / 4$} \\
\hline$m$ & $f_{m}$ & $g_{m}$ \\
\hline basic & $+0.13640434(+1)$ & \\
\hline $\begin{array}{l}0 \\
1 \\
2 \\
3 \\
4\end{array}$ & $\begin{array}{l}+0.94335081(+0) \\
+0.47094227(+0) \\
+0.15962976(+0) \\
+0.52222212(-1) \\
+0.17272056(-1)\end{array}$ & $\begin{array}{l}-0.37183474(+0) \\
-0.18563192(+0) \\
-0.97767116(-1)\end{array}$ \\
\hline $\begin{array}{l}5 \\
6 \\
7 \\
8 \\
9\end{array}$ & $\begin{array}{l}+0.55233089(-2) \\
+0.16203994(-2) \\
+0.41694492(-3) \\
+0.90138919(-4) \\
+0.15575188(-4)\end{array}$ & 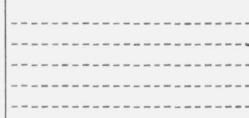 \\
\hline $\begin{array}{l}10 \\
11 \\
12\end{array}$ & $\begin{array}{l}+0.20048427(-5) \\
+0.17022684(-6) \\
+0.71319103(-8)\end{array}$ & 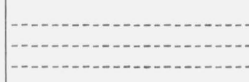 \\
\hline $\begin{array}{l}\text { Equator } \\
\text { check }\end{array}$ & \multicolumn{2}{|c|}{$\begin{array}{c}B_{\rho} / B \sin \phi=-0.99999999 \\
\Delta \rho / a: \text { negligible }\end{array}$} \\
\hline $\begin{array}{l}\text { Pole } \\
\text { check... }\end{array}$ & \multicolumn{2}{|c|}{$\begin{aligned} a \frac{\partial}{\partial \rho}\left(B_{z} / B \sin \phi\right) & =+0.11001902 \\
\Delta z / b & =+0.01246495\end{aligned}$} \\
\hline
\end{tabular}

TABLE 2

\begin{tabular}{|c|c|c|}
\hline \multicolumn{3}{|c|}{$a / b=1 / 2$} \\
\hline$m$ & $f_{m}$ & $g_{m}$ \\
\hline basic & $+0.13390263(+1)$ & \\
\hline $\begin{array}{l}0 \\
1 \\
2 \\
3 \\
4\end{array}$ & $\begin{array}{l}+0.76790238(+0) \\
+0.23079152(+0) \\
+0.58147254(-1) \\
+0.16764875(-1) \\
+0.50092196(-2)\end{array}$ & $\begin{array}{l}-0.32481666(+0) \\
-0.14321008(+0) \\
-0.97128394(-1) \\
-0.67455522(-1) \\
-0.36913235(-1)\end{array}$ \\
\hline $\begin{array}{l}5 \\
6 \\
7 \\
8 \\
9\end{array}$ & $\begin{array}{l}+0.13805064(-2) \\
+0.32120205(-3) \\
+0.57675668(-4) \\
+0.70016001(-5) \\
+0.42707647(-6)\end{array}$ & $-0.11074155(-1)$ \\
\hline $\begin{array}{l}\text { Equator } \\
\text { check }\end{array}$ & \multicolumn{2}{|c|}{$\begin{array}{c}B_{\rho} / B \sin \phi=-1.00000004 \\
\Delta \rho / a: \text { negligible }\end{array}$} \\
\hline $\begin{array}{l}\text { Pole } \\
\text { check. }\end{array}$ & \multicolumn{2}{|c|}{$\begin{aligned} a \frac{\partial}{\partial \rho}\left(B_{z} / B \sin \phi\right) & =-0.00614087 \\
\Delta z / b & =-0.02256388\end{aligned}$} \\
\hline
\end{tabular}

TABLE 3

\begin{tabular}{|c|c|c|}
\hline \multicolumn{3}{|c|}{$a / b=1$} \\
\hline$m$ & $f_{m}$ & $g_{m}$ \\
\hline basic & $+0.12992927(+1)$ & \\
\hline $\begin{array}{l}0 \\
1 \\
2 \\
3 \\
4\end{array}$ & $\begin{array}{l}+0.54323936(+0) \\
+0.92098198(-1) \\
+0.17619634(-1) \\
+0.38217146(-2) \\
+0.67793221(-3)\end{array}$ & $\begin{array}{l}-0.29402467(+0) \\
-0.12410492(+0) \\
-0.80893285(-1) \\
-0.59953649(-1) \\
-0.44063915(-1)\end{array}$ \\
\hline $\begin{array}{l}5 \\
6 \\
7 \\
8 \\
9\end{array}$ & $\begin{array}{c}+0.66769575(-4) \\
-\end{array}$ & $\begin{array}{l}-0.29238560(-1) \\
-0.16182197(-1) \\
-0.68464716(-2) \\
-0.19415309(-2) \\
-0.27441144(-3)\end{array}$ \\
\hline $\begin{array}{l}\text { Equator } \\
\text { check.-- }\end{array}$ & \multicolumn{2}{|c|}{$\begin{array}{c}B_{\rho} / B \sin \phi=-0.99999998 \\
\Delta \rho / \alpha: \text { neglible }\end{array}$} \\
\hline $\begin{array}{l}\text { Pole } \\
\text { check.-- }\end{array}$ & \multicolumn{2}{|c|}{$\begin{aligned} a \frac{\partial}{\partial \rho}\left(B_{z} / B \sin \phi\right) & =-0.00000485 \\
\Delta z / b & =-0.00001512\end{aligned}$} \\
\hline
\end{tabular}

Table 4

\begin{tabular}{|c|c|c|}
\hline \multicolumn{3}{|c|}{$a / b=2$} \\
\hline$m$ & $f_{m}$ & $g_{m}$ \\
\hline basic & $+0.12407197(+1)$ & 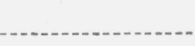 \\
\hline $\begin{array}{l}0 \\
1 \\
2 \\
3 \\
4\end{array}$ & $\begin{array}{l}+0.33590275(+0) \\
+0.30150397(-1) \\
+0.28485520(-2) \\
\end{array}$ & $\begin{array}{l}-0.25652350(+0) \\
-0.11210208(+0) \\
-0.72413481(-1) \\
-0.52386398(-1) \\
-0.37934754(-1)\end{array}$ \\
\hline $\begin{array}{l}5 \\
6 \\
7 \\
8 \\
9\end{array}$ & 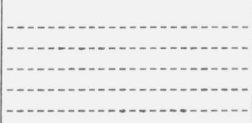 & $\begin{array}{l}-0.25696044(-1) \\
-0.15530579(-1) \\
-0.80562157(-2) \\
-0.34498633(-2) \\
-0.11632626(-2)\end{array}$ \\
\hline $\begin{array}{l}10 \\
11 \\
12\end{array}$ & 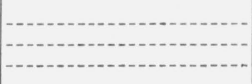 & $\begin{array}{l}-0.28842547(-3) \\
-0.46632080(-4) \\
-0.36819245(-5)\end{array}$ \\
\hline $\begin{array}{c}\text { Equator } \\
\text { check }\end{array}$ & \multicolumn{2}{|c|}{$\begin{aligned} B \rho / B \sin \phi & =-1.00000288 \\
\Delta \rho / a & =+0.00000115\end{aligned}$} \\
\hline $\begin{array}{l}\text { Pole } \\
\text { check_-- }\end{array}$ & \multicolumn{2}{|c|}{$\begin{aligned} a \frac{\partial}{\partial \rho}\left(B_{z} / B \sin \phi\right) & =-0.00000013 \\
\Delta z / b & =-0.00000100\end{aligned}$} \\
\hline
\end{tabular}

TABLE 5 
TABle 6

\begin{tabular}{|c|c|c|}
\hline \multicolumn{3}{|c|}{$a / b=4$} \\
\hline$m$ & $f_{m}$ & $g_{m}$ \\
\hline basic & $+0.11775253(+1)$ & \\
\hline $\begin{array}{l}0 \\
1 \\
2 \\
3 \\
4\end{array}$ & $\begin{array}{r}+0.18232541(+0) \\
+0.76983950(-3) \\
\end{array}$ & $\begin{array}{l}-0.21277158(+0) \\
-0.98721317(-1) \\
-0.61346284(-1) \\
-0.39649681(-1) \\
-0.24392554(-1)\end{array}$ \\
\hline $\begin{array}{l}5 \\
6 \\
7 \\
8 \\
9\end{array}$ & 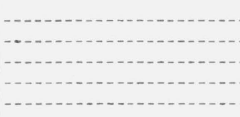 & $\begin{array}{l}-0.13622852(-1) \\
-0.66941775(-2) \\
-0.28211792(-2) \\
-0.99369454(-3) \\
-0.28363133(-3)\end{array}$ \\
\hline $\begin{array}{l}10 \\
11 \\
12 \\
13\end{array}$ & (n-n & $\begin{array}{l}-0.62898095(-4) \\
-0.10154428(-4) \\
-0.10608227(-5) \\
-0.53798542(-7)\end{array}$ \\
\hline $\begin{array}{l}\text { Equator } \\
\text { check }\end{array}$ & \multicolumn{2}{|c|}{$\begin{aligned} B \rho / B \sin \phi & =-1.00378633 \\
\Delta \rho / a & =+0.00094822\end{aligned}$} \\
\hline $\begin{array}{l}\text { Pole } \\
\text { check_. }\end{array}$ & \multicolumn{2}{|c|}{$\begin{array}{r}a \frac{\partial}{\partial \rho}\left(B_{z} / B \sin \phi\right)=+0.00000000 \\
\Delta z / b: \text { negligible }\end{array}$} \\
\hline
\end{tabular}

\section{References}

[1] T. T. Taylor, Electric polarizability of a short right circular conducting cylinder, J. Research NBS 64B3, 135 (1960).

[2] W. R. Smythe, Static and dynamic electricity, 2d ed. (McGraw-Hill Book Co., Inc., New York. N.Y., 1950).

[3] A. Erdélyi, Tables of integral transforms II (Bateman Manuscript Project, McGraw-Hill Book Co., Inc., New York, N.Y., 1954).

[4] A Erdélyi, Higher transcendental functions I (Bateman Manuscript Project, McGraw-Hill Book Co., Inc., New York, N.Y., 1953).

(Paper 64B4-35) 